\title{
Resemblance and Mergence based Indexing for High Performance Data Deduplication
}

\author{
Panfeng Zhang ${ }^{\mathrm{a}}$, Ping Huang ${ }^{\mathrm{b}}$, Xubin He $\mathrm{He}^{\mathrm{b}, \mathrm{c}}$, Hua Wang ${ }^{\mathrm{a}, *}, \mathrm{Ke}_{\text {Zhou }}{ }^{\mathrm{a}}$ \\ ${ }^{a}$ School of Computer, Huazhong University of Science and Technology, Wuhan, China \\ Wuhan National Laboratory for Optoelectronics, Wuhan, China \\ ${ }^{b}$ Department of Computer and Information Sciences, Temple University, USA \\ ${ }^{c}$ Department of Electrical and Computer Engineering, Virginia Commonwealth University, USA
}

\begin{abstract}
Data deduplication, a data redundancy elimination technique, has been widely employed in many application environments to reduce data storage space. However, it is challenging to provide a fast and scalable key-value fingerprint index particularly for large datasets, while the index performance is critical to the overall deduplication performance. This paper proposes RMD, a resemblance and mergence based deduplication scheme, which aims to provide quick responses to fingerprint queries. The key idea of RMD is to leverage a bloom filter array and a data resemblance algorithm to dramatically reduce the query range. At data ingesting time, RMD uses a resemblance algorithm to detect resemble data segments and put resemblance segments in the same bin. As a result, at querying time, it only needs to search in the corresponding bin to detect duplicate content, which significantly speeds up the query process. Moreover, RMD uses a mergence strategy to accumulate resemblance segments to relevant bins, and exploits frequency-based fingerprint retention policy to cap the bin capacity to improve query throughput and data deduplication ratio. Extensive experimental results with real-world datasets have shown that RMD is able to achieve high query performance and outperforms several well-known deduplication schemes.
\end{abstract}

Keywords: fast index, deduplication, resemblance mergence, fingerprint retrieval, key value index.

\section{Introduction}

With the rapid development of information technology, a tremendous amount of data has been generated and data is being generated at an accelerating rate. As a space-efficient technology for reducing storage overhead, deduplication has attracted a great deal of attention and popularity in various storage systems, such as primary storage systems (Chen and Shen, 2016a), secondary backup systems (Kaczmarczyk and Dubnicki, 2015; Amvrosiadis and Bhadkamkar, 2015; Li et al., 2014), cloud storage systems (Li et al., 2015; Fu et al., 2014; Mao et al., 2014) and virtual machine systems (Xu et al., 2016). Deduplication, as a global data redundancy removal technology, mainly identifies

\footnotetext{
${ }^{*}$ Corresponding author

Email addresses: panf_zhang@hust.edu.cn (Panfeng Zhang), templestorager@temple.edu (Ping Huang), xubin.he@temple.edu (Xubin He), hwang@hust. edu.cn (Hua Wang ), k.zhou@hust.edu.cn (Ke Zhou )
}

Preprint submitted to Journal of Systems and Software duplicate data content, stores only one data copy, and replaces other identical copies with indirected references rather than storing full copies. Typically, data deduplication relies on fingerprinting to find duplicate data instead of using byte-by-byte comparison. In deduplication systems, a fingerprint of a file or chunk is calculated using a cryptographic digest algorithm (e.g. SHA-1). Fingerprints serve as the proxies for testing content uniqueness. A fingerprint index has to be established for mapping fingerprints to the physical addresses of files or chunks. A duplicate file or chunk can be identified via checking the existence of its fingerprint in the fingerprint index. Because the probability of hash collision is much smaller than that of hardware errors (Quinlan and Dorward, 2002), hash-based fingerprint index is realistically widely used in real-world storage and backup systems.

Methods of detecting duplicate fingerprints fall into two categories (Fu et al., 2015). One is exact detection which examines every freshly arriving fingerprint against the whole fingerprint index. This strategy aims

December 19, 2016 
to find out all duplicate data at the cost of exhaustively checking all fingerprints in the index store. A critical shortcoming with this method is that it suffers from fingerprint query performance as it may incur excessive disk accesses due to the large search space. The other one is near-exact detection in which every fingerprint only checks a portion of the fingerprint index at the risk of losing some deduplication opportunities, achieving approximate deduplication ratio, i.e., the ratio between the original total data size and the amount of actually stored data after deduplication, which represents data deduplication efficiency and is an important indicator for comparing different deduplication systems.

In recent years, data-intensive applications have become popular in cloud environments(Shvachko et al., 2010; Maltzahn et al., 2010), resulting in a huge amount of data having been generated. Data deduplication has been leveraged to facilitate the management of such "big data". One of the main challenges facing data deduplication is to provide a high performance and scalable key-value fingerprint index for deduplicating largescale datasets (Debnath et al., 2010). For example, to support a unique dataset of $800 \mathrm{~TB}$ and assuming an average chunk size of $8 \mathrm{~KB}$, at least $2 \mathrm{~TB}$ of SHA1(20-byte) fingerprints will be generated, which are too large to be stored in memory (Xia et al., 2015). Therefore, in practice, the entire fingerprint index is stored either in disk system (Min et al., 2011) or on flash (Debnath et al., 2010), with part of the fingerprints cached in a memory buffer. However, disk or flash access speed is much slower than that of memory, which creates the well-known fingerprint bottleneck problem (Zhu et al., 2008). Fortunately, due to the existence of locality, in most cases it only requires to check against a portion of the fingerprints using near-exact deduplication, without significantly losing deduplication efficiency.

Our goal is to design an efficient fingerprint index store which can provide high query performance, while minimally sacrificing deduplication efficiency. The normal fingerprint index implemented using B-tree or hash table cannot meet efficient fingerprint query requirements from massive fingerprints. How to cope with this bottleneck has become one of the major challenges in deduplication systems. To this end, we propose RMD, a new resemblance and mergence based near-exact deduplication scheme, which can provide very high index query performance by reducing the search space via resemblance-based segment store organization. The key idea of RMD is to exploit the data resemblance theory and bloom filter array to narrow the query range. Specifically, RMD uses the resemblance algorithm to detect resemblance segments and clusters resemblance segments into the same bin. To deduplicate a newly incoming segment, RMD first quickly locates the bin to which the new segment resembles and then checks the chunks in the segment against all the chunks in that bin for deduplication opportunities. To speed up the procedure of finding the appropriate bin, particularly in the case of extremely large number of fingerprints, RMD leverages a bloom filter array which is a high performance scalable data structure suitable for membership query. Due to the clustering of the resemblance segments, RMD can rapidly find all the potential segments for comparisons to identify duplicate chunks, which might be distributed in a large index space with other existing deduplication approaches. Overall, with the employment of bloom filter array and clustering of resemblance segments, RMD can quickly locate as many resemblance segments as possible for deduplication, which significantly reduces disk I/O accesses incurred by fingerprint queries, thus improving deduplication performance.

In summary, our contributions in this paper include:

- We propose a new deduplication scheme called RMD which leverages a new two-level fingerprint index structure. It combines the data resemblance theory and the DBA algorithm to ensure high fingerprint query performance. Data resemblance algorithm is used to identify and cluster resemblance segments into the same bin so as to significantly reduce the index space. The bloom filter array is used to speed up the procedure of locating the right bin

- We exploit segment based resemblance approach to indexing duplicate data, and test the best segment cardinality. In doing that, RMD improves index performance effectively and overcomes the problems associated with file based resemblance approaches.

- We propose a resemblance mergence strategy that classifies and merges segments into distinct bins according to their representative fingerprints. Fingerprint bins collect duplicate fingerprints from resemblance segments of the data stream, which increases the data deduplication efficiency. Fingerprint bins can be read and written as a whole when accessing memory and disk, which increases fingerprint retrieval efficiency and overcomes the problems of fragmented storage of resemblance segments. 
- We explore the distribution of fingerprint frequency and propose a frequency-based clean-up strategy to eliminate low-frequency fingerprints in Bins. Doing so significantly reduces the fingerprint bin sizes at the cost of a marginal impact on the deduplicaton ratio.

- We have implemented a prototype system of RMD and performed comprehensive evaluations with real-world datasets to evaluate RMD. The evaluation results have shown that RMD can provide exceptionally good fingerprint index performance with minimal RAM consumption due to quickly locating the chunk comparison candidates. Moreover, RMD outperforms several other deduplication approaches in terms of fingerprint query performance.

The rest of the paper is organized as follows. Section 2 presents background and motivation for this work. Section 3 describes the architecture and design details of the proposed RMD deduplication approach. Section 4 presents our experimental results and evaluations. Section 5 discusses the related work. Finally, Section 6 concludes our paper.

\section{Background and Motivation}

In this section, we first provide the necessary background knowledge for RMD, and then motivate our work by analyzing the observations about resemblance based deduplication approaches using real-world workloads.

\subsection{Challenges of Fingerprint Index}

The fingerprint index is a well-recognized performance bottleneck in large-scale deduplication systems. There are a large body of work addressing the index bottleneck problem in previous literature (Zhu et al., 2008; Debnath et al., 2010). One approach to organizing the fingerprint index is to use a key-value index table (Shim, 2017; Wang et al., 2013), with the key being a fingerprint and the value indicating the physical address where the corresponding file or chunk is located. A duplicate file or chunk is identified by checking the existence of its fingerprint in the key-value index table. As pointed out in previous research (Dong et al., 2011), two primary factors affect the fingerprint index performance. One factor is the size of fingerprint index table, which could be too large to be completely kept in RAM. As a result, the entire fingerprint index is typically backed up in the disk or flash storage with a portion of fingerprints being kept in a memory buffer. However, since the fingerprint access is completely random in nature, using an HDD disk system to store the fingerprint index creates a performance bottleneck due to the slow random access HDDs, particularly in largescale systems with huge amounts of fingerprints. The other one is the required computing resources, which could impose non-trivial overheads on the backup performance.

In exact deduplication schemes, a major challenge is how to quickly compare chunks against the entire fingerprint index. To realize that, practically, designers choose to store the entire fingerprint index in disk systems, and employ optimization techniques to remedy the lower performance of disk systems (Fu et al., 2015). For example, the DDFS (Zhu et al., 2008) achieves exact deduplication using Summary Vector and data prefetching to speed up fingerprint index queries. PRUNE (Min et al., 2011) exploits temporal locality and Context Aware Chunking to build an exact deduplication system, while ChunkStash (Debnath et al., 2010) stores the fingerprint index on high performance flashbased storage to satisfy fingerprint queries.

In contrast to exact deduplication, near-exact deduplication avoids exhaustively comparing against the entire fingerprint index at the cost of slightly sacrificing deduplication efficiency. For an incoming segmen$t$, it compares only part of fingerprints. Typically, near-exact deduplication employs multiple techniques to improve performance, such as prefetching, caching, sampling (Bianco et al., 2015), etc. Spatial locality has been leveraged to enable efficient disk data prefetching (Srinivasan et al., 2012) to speed up index queries. Whereas, data temporal locality has been exploited to help find duplicate data (Wildani et al., 2013; Min et al., 2011). Sampling strategies (Lillibridge et al., 2009) have been used to locate high probability area of duplicate data. A sampling strategy cuts data streams into consecutive segments each of which contains hundreds or thousands of chunks, and for each segment it samples a representative chunk. It then uses the representative fingerprint to identify and locate relevant segments. There are two commonly used sampling strategies, uniform chunk sampling (Debnath et al., 2010) and minimum chunk sampling (Bhagwat et al., 2009). Uniform chunk sampling selects the $i^{\text {th }}$ chunk in every segment as its representative chunk and minimum chunk sampling chooses the chunk which has the minimum fingerprint as its representative chunk. Our proposed RMD leverages sampling techniques and a resemblance algorithm to identify resemblance segments and group them into the same bin. 


\subsection{Resemblance Algorithm}

Data resemblance theory (Broder, 1997, 2000) has been used for searching identical text blocks among different documents. According to Broder's theorem:

Theorem 1: Considering two sets S1, S2, and assume $\mathrm{H}(\mathrm{S} 1)$ and $\mathrm{H}(\mathrm{S} 2)$ are the hashed fingerprint sets corresponding to the elements in S1 and S2, respectively. Let $\min (\mathrm{H}(\mathrm{S}))$ denote the smallest value of $\mathrm{H}(\mathrm{S})$. Then:

$$
\operatorname{Pr}[\min (H(S 1))=\min (H(S 2))]=\frac{|S 1 \cap S 2|}{|S 1 \cup S 2|}
$$

The above theory states that, when the minimum hash value of the elements in one set equals to that of the other set, there exists a good probability that the two sets share certain number of elements. In the context of deduplication systems, the theory means, if the two chosen minimum fingerprints from two different fingerprint sets are the same, then there exists a high probability that the two fingerprint sets share great commonality. For the convenience of discussion, the minimum fingerprint in a set is named representative fingerprint of the set (hereinafter referred to as $R F$ ). Many near-exact deduplication systems leverage this theorem to find the fingerprint candidate sets for comparisons to narrow down fingerprint indexing space (Bhagwat et al., 2009; Lillibridge et al., 2009; Xia et al., 2015).

In a deduplication system, the directories and files of an incoming dataset are scanned to form a list of files. Each file in the list is divided into fix-sized chunks using fixed-sized length segmenting mechanisms or into variable-sized chunks in a content-defined manner (Kruus et al., 2010). A fingerprint is computed for each chunk, forming a fingerprint list. Fig. 1 shows an example to illustrate how to obtain the fingerprint list and how to apply data resemblance theory.

According to the data resemblance theory, a fingerprint stream (list) has to be divided into multiple fingerprint subsets. A resultant subset is defined as a segment in this paper, and in the fingerprint index table, this subset is stored in the same Fingerprint Bin. In the following, we discuss several previous works which leverage the resemblance theorem.

Extreme binning (Bhagwat et al., 2009) is a good example of introducing minimum chunk sampling to a deduplication system. In the system, the whole file is used as the basic resemblance unit and therefore its design is only effective for part of datasets. On one hand, for a dataset with lots of large size files, both the retrieval performance and deduplication ratio are poor. On the other hand, for a dataset with dominating small size files, the retrieval performance is poor, though the deduplication ratio is good.

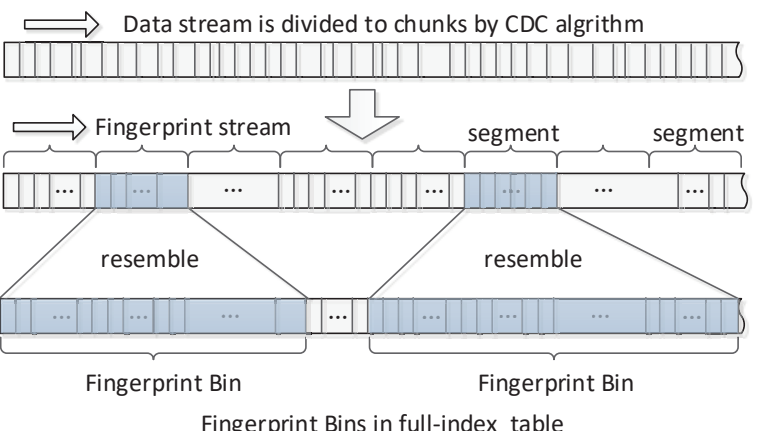

Figure 1: An illustrative example showing how to get a fingerprint list and how to apply data resemblance theory. The data stream is divided into chunks by a CDC algrithm, resulting in a chunk list. A fingerprint list is then obtained via computing the fingerprints of the chunks. For each coming segment, it is only necessary to examine the resemblance fingerprint bin to find duplicate data, avoid searching the full-index table.

Sparse indexing (Lillibridge et al., 2009) applies multi-segment prefetching strategy as well as resemblance algorithm to realize data deduplication, which effectively reduces RAM requirements and improves deduplication ratio. However, to deduplicate an incoming segment, it requires several rounds of comparisons.

SiLo (Xia et al., 2015) combines the merits of both Extreme binning and Sparse Indexing (Lillibridge et al., 2009). It analyzes the resemblance based theory and suggests that the resemblance probability can be increased by partitioning datasets to small segments.

\subsection{Resemblance Segment Mergence in RMD}

Our proposed RMD aims to absorb the respective advantages of previous schemes, while avoiding their drawbacks, to achieve better fingerprint query performance. One characteristic of RMD is that it adopts segment mergence policy, i.e., it compacts resemblance segments to one Fingerprint Bin, so that the process of identifying the resemblance segments for deduplication can be greatly accelerated.

RMD classifies and merges resemblance segments in fingerprint streams to different bins according to their RFs. A RF index table is maintained for mapping RFs to the Fingerprint Bin addresses and each RF corresponds to one bin. By doing so, RMD can quickly ascertain the bin location to get the resembling fingerprint bin for comparison instead of looking for a large spectrum of space to search for dispersed segments, which greatly shrinks the search scope and speeds up the deduplication procedure. 


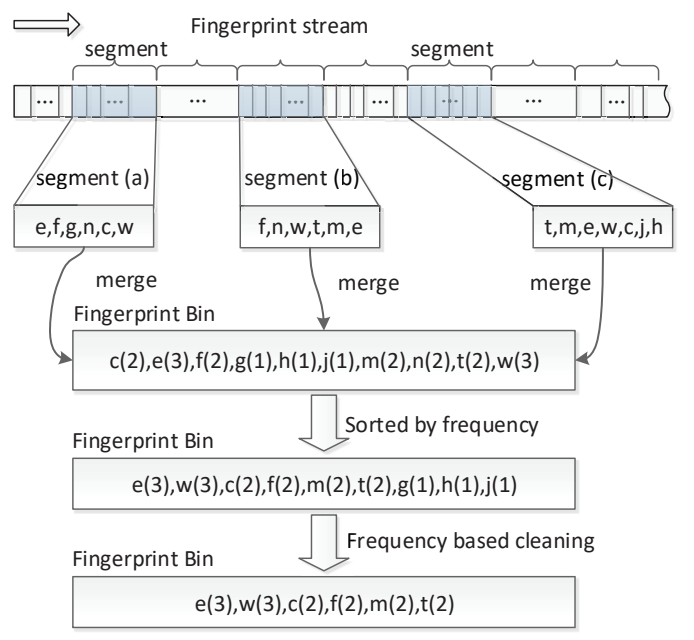

Figure 2: Resemblance segment mergence and frequency based placement. Resemblance segments (a), (b), and (c) are merged into one fingerprint bin. After mergence, the fingerprints in the bin are sorted according to their frequency. Less frequent fingerprints are dropped off if capacity is limited.

Fig. 2 shows an example illustrating how resemblance segment mergence works. In the figure, we assume there are three segments in a fingerprint stream. Segment (a) contains $[e, f, g, n, c, w]$, segment (b) contains $[f, n, w, t, m, e]$, and segment (c) contains $[t, m, e, w, c, j, h]$, where each char represents a chunk fingerprint. The three segments are resemblance segments as they have the same representative fingerprint, which is $e$. Knowing that the three segments are resemblance segments, RMD compacts them together and stores them in one location, namely a Fingerprint Bin. When a new segment having the same representative fingerprint $e$ arrives, RMD compares all of its fingerprints against the fingerprints in the resemblance bin and it is very likely that most duplicate chunks can be successfully identified due to locality. Overall, merging resemblance segments can reduce the number of disk IOs incurred by fingerprints examinations, while improving the accuracy of locating duplicate fingerprint candidates.

\subsection{Frequency Based Buffer Strategy}

It is well-known that duplicate data exhibit both temporal and spatial locality (Srinivasan et al., 2012). Besides, duplicate data also exhibit frequency characteristic, which means different data chunks have different occurrences. Some chunks exhibit higher occurrences, e.g., duplicate chunks, while others show smaller repetitions, e.g., unique chunks. We examine real-world datasets to investigate their frequency

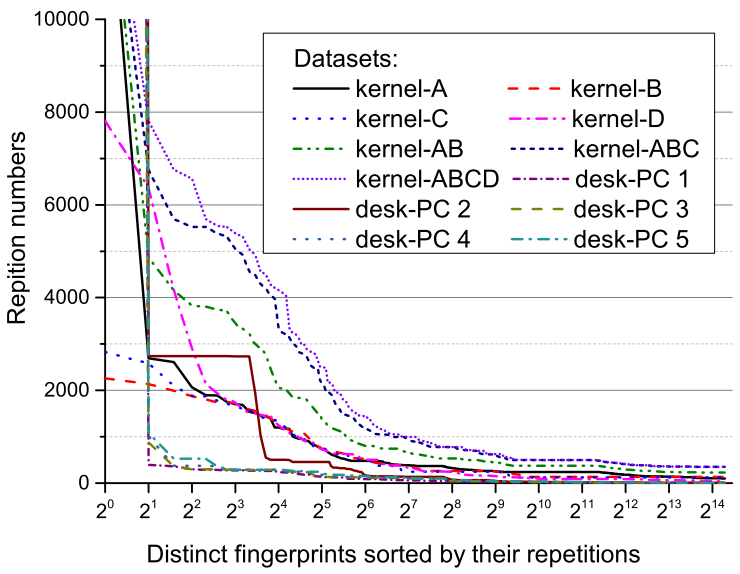

Figure 3: The fingerprint repetition distribution of 9 datasets, including 4 Linux kernel source datasets and 5 daily lab desk-PC datasets. We have also created Linux dataset combinations, e.g., kernel-AB indicates the dataset combining kernel-A and kernel-B. The $\mathrm{x}$-axis denotes the fingerprint sequence in descending order and the $y$-axis represents the repetition numbers of the corresponding fingerprints. It shows fingerprints exhibit skewness, which validates our frequency based buffer strategy.

patterns. Fig. 3 shows the fingerprint repetitions of 9 datasets, including 4 Linux kernel sources downloaded from https://www.kernel.org, and 5 desk-PC datasets from our lab students' desk-PC backups. The details of datesets are shown in Table 1. From the figure, we can clearly see that chunks exhibit highly skewed frequency patterns. Some chunks appear much more frequently than others, while other chunks have very low repetitions. A reasonable speculation could be that the more frequently a fingerprint appears, the more likely it will appear again in the future. For example, the same fingerprints will appear again and again across consecutive backups if the content has no change. Therefore, the findings indicate that if memory space is limited we can keep only the most frequent fingerprints in the buffer without losing noticeable deduplication efficiency.

\subsection{DBA and its scalability}

A bloom filter array is a high performance and scalable data structure, which can be used for quickly answering membership queries and decreasing index space to speed up index process. It typically contains multiple homogeneous bloom filters, which share a set of hash functions but not bit vectors. All bit vectors constitute a vector matrix, in which each row bit vector represents a bloom filter. Bit vectors can operate either serially or in parallel. 
Table 1: Features of the used datasets

\begin{tabular}{rrrrr}
\hline datasets & Total size (GB) & Total Files & Total Chunks & Exact-Deduplication Ratio \\
\hline Kernel-A & 52.10 & 4464198 & 13763497 & 16.89 \\
Kernel-b & 48.65 & 4160274 & 12758277 & 45.53 \\
Kernel-c & 52.57 & 4587572 & 13801916 & 49.62 \\
Kernel-d & 26.94 & 1063982 & 6063052 & 4.40 \\
Desk-PC1 & 12.30 & 54377 & 2690726 & 1.27 \\
Desk-PC2 & 10.76 & 77273 & 2731045 & 1.33 \\
Desk-PC3 & 7.21 & 41381 & 1682440 & 1.71 \\
Desk-PC4 & 9.78 & 61873 & 2361114 & 1.69 \\
Desk-PC5 & 2.27 & 15730 & 542294 & 1.33 \\
\hline
\end{tabular}

As a deduplication system ages, data accumulates and the total amount of stored data can grow to be extremely large, causing scalability problem. Particularly, a high performance and scalable fingerprint index store is critically important to a scalable deduplication system. Due to its excellent expandability, the bloom filter array has been used in deduplication systems to implement fast fingerprint queries. Existing bloom filter array schemes, such as dynamic Bloom Filters (DBF) (Guo et al., 2006), scalable Bloom filters (Xie et al., 2007; Almeida et al., 2007), and incremental Bloom Filters (Hao et al., 2008) have their limitations when datasets scale up. A DBF suffers the scalability problem if it contains too many bloom filters. Considering a DBF using $k$ hash functions and $r$ Bloom Filters, the time complexity of querying a fingerprint is $O(k \times r)$, which increases linearly with the number of bloom filters. Fig. 4 shows the query performance trend as the number of bloom filter increases (Wei et al., 2014). At the initial phase, an amount of $2^{19}$ fingerprints are inserted into one bloom filter and as more fingerprints are inserted, the number of bloom filters increases. When 128 bloom filters are created, the query performance drops to only $32 \%$ of the initial performance, illustrating that DBF is not scalable in fingerprint query services.

To overcome the scalability limitation of DBF, Dynamic Bloom filter array (DBA) (Wei et al., 2014), an innovative bloom filter array was proposed. DBA is based on existing bloom filter array and uses multiple bloom filter parallel detection algorithm rather than serial detection algorithm as in conventional DBF. Therefore, DBA has excellent retrieval performance and scalability. Our RMD employs a DBA as a frontend module to speed up fingerprint queries and provide scalability.

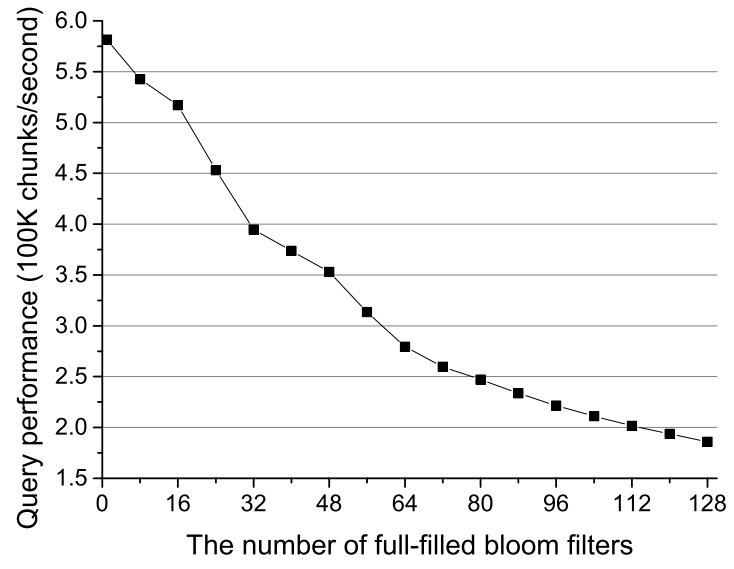

Figure 4: The query performance trend of $\mathrm{DBF}$ as more fingerprints are loaded and more Bloom filters are dynamically created (Wei et al., 2014). As it shows, when 128 bloom filters have been created, the query performance drops to only $32 \%$ of the initial phase when only one bloom filter is in place, demonstrating that DBF has severe scalability problem.

\section{Design and Implementation}

In this section, we discuss the design and implementation details of RMD. We first present an overview of RMD, and then discuss the key data structures that support the high performance of RMD, followed by descriptions of the employed techniques. Finally, we give a summary of the overall workflow of RMD and discuss the system scalability.

\subsection{The Fingerprint Index in RMD}

RMD is based on two main techniques, which are resemblance detection and dynamic bloom filter array, to provide fast fingerprint queries. The key differences between RMD and other near exact deduplication approaches are two-fold. First, RMD introduces a novel on-disk fingerprint organization by clustering resemblance segments sharing the same representative 


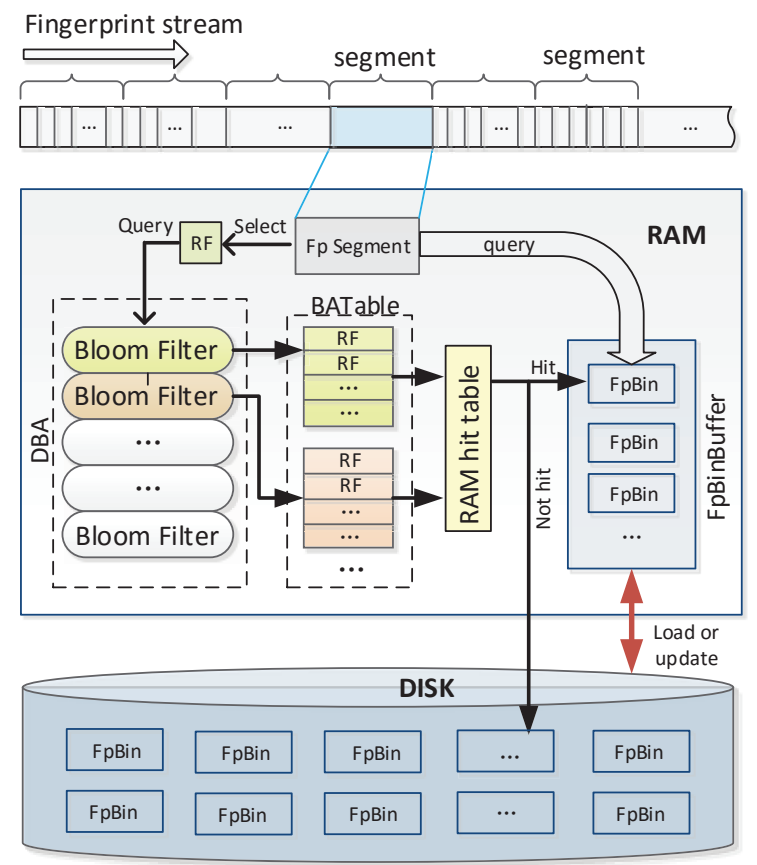

Figure 5: The overview of RMD structure. RMD has five main components, DBA, BATable, RAM hit table, FpBinBuffer, and ondisk FpBin factory. The representative fingerprint (RF) of a segment is used to locate resemblance segments via consulting DBA and BATable. Once RMD finds the fingerprint bin, the whole bin is brought into memory and buffered in the FpBinBuffer fingerprint comparisons

fingerprint (RF) into one bin which can be brought into memory with a single disk operation. Conventional systems may need multiple disk operations to fetch those resemblance segments, as they are dispersed in different locations. Second, RMD employs the high performance and scalable DBA as a front-end module to speed up finding the corresponding segment bin. The deployment of those two techniques ensures that RMD delivers high fingerprint query performance and features good scalability for accommodating increasing datasets.

Fig. 5 gives an overview of the RMD structure, consisting of five main components: Dynamic Bloom Filter Array (DBA), Bin Address Tables (BATable), Fingerprint Bin Buffer (FpBinBuffer), RAM hit table, and on-disk Fingerprint Bins (FpBin). The DBA and Bin Address Table (BATable) work together to find the corresponding fingerprint bin given a RF. Each fingerprint bin contains fingerprints from resemblance segments sharing the same RF and the FpBinBuffer is used to buffer fingerprint bins. In the following, we discuss each of the components in more details.

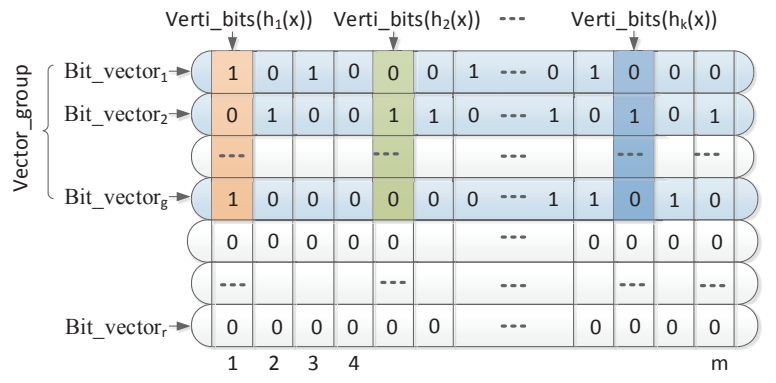

Figure 6: The structural overview of DBA's bit vectors. Every $g$ bit vectors form a Vector_group, and the bits locating at the same column positions of the vectors in a group constitute a Verti_bits. Vectors in the same group can operate in parallel to provide high performance.

\subsection{Dynamic Bloom Filter Array (DBA)}

RMD uses the DBA to facilitate RF queries. Each bloom filter in the DBA is associated with one bin address table as shown in Fig. 5. Every $g$ bloom filters form a group. Assume the DBA has $r$ bloom filters, then the number of groups is $\lceil\mathrm{r} / \mathrm{g}\rceil$. In our DBA implementation, we choose to use the same set of hash functions across all bloom filters for simplicity and the parameters of bit vectors of the bloom filters in one group are set to be the same, while they are different across groups. Bloom filters in the same group can operate in parallel, while bloom filters in different groups need to operate serially, as only one group can be in effect at any given time.

Fig. 6 shows the structural view of the DBA's bit vectors. All bit vectors form a bit matrix, which is used for representative fingerprints query. Every $g$ homogeneous bit vectors constitute a Vector_group and the bits locating at the same column positions of the bit vectors comprise a Verti_bits as highlighted in the figure. All Verti_bits in the same group can operate simultaneously to leverage parallelism. The value of $g$ is typically set to the machine word length.

Fig. 7 gives an example demonstrating how RF $x$ is queried in parallel within each bloom filter group. First, the $k$ independent hash functions $\left(h_{i}(x)\right)$ are computed and the $k$ values are used as the offsets of bit vectors in the group. According to the $k$ hash values, we can obtain the $k$ Verti_bits. Second, the $k$ Verti_bits are ANDed and the result will convey the information regarding whether RF $x$ exists or not. If the result equals to zero, then RF $x$ is assumed not to exist in the Vector_group. If the result is not zero, then according to the offset of bit 1, we can locate the bit vector and thus find the corresponding Bin Address Table. The same query process is repeated in other vector groups until the bin address table are found or all vector groups have been examined which indicates 


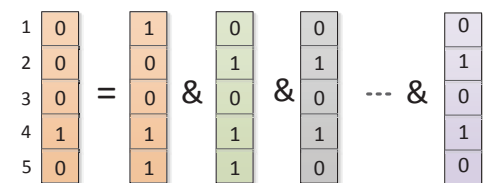

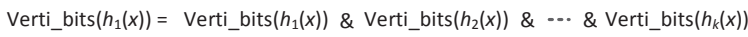

Figure 7: This figure illustrates the query process of a RF $x$. The $k$ hash functions are first calculated and then the resultant $k$ Verti_bits are ANDed. The final result of the AND operation reveals the existence of RF $x$.

RF $x$ is a new representative fingerprint and need to be inserted into the DBA.

From the above discussion, we know that DBA's query mechanisms are quite different from previous bloom filter arrays, which typically perform queries serially as opposed to in parallel. However, it should be noted that though bloom filter is an efficient data structure, it may cause the potential "false positive" problem. More specifically, bloom filters can affirmatively answer queries like "RF $x$ is not in a set", but it cannot positively answer "RF $x$ is in a set". Fortunately, the probability of false positive can be controlled by adjusting the relevant bloom filter parameters (Wei et al., 2014).

In RMD, when the front-end DBA gives a positive answer about the existence of a RF, the RF needs to be further searched in the Bin Address Table, where "false positive" is eliminated if it is found in the Bin Address Table as discussed in the next subsection.

\subsection{Bin Address Table}

The Bin Address Table is a mapping table composed of RFs and Fingerprint Bin Addresses. It takes on three roles, namely, RF index, false positive verification, as well as mapping RFs to the addresses of Fingerprint Bins. Fig. 8 shows the organization of a Bin Address Table. Each bloom filter is responsible for a subset of RFs and each RF corresponds to a fingerprint bin which consists of many fingerprints from resemblance segments. All fingerprint bins constitute the entire fingerprint index space. The Bin Address Table stores complete RFs. Searching for a RF in the Bin Address Table follows two steps which is shown in Fig. 9. First, a checking process is performed on the frontend DBA and the checking result will tell whether the $\mathrm{RF}$ is in the Bin Address Table or not. Second, based on the query result, RMD continues to either create a new Bin Address Table entry or search for the RF in the Bin Address Table. If the DBA gives a negative answer, then the RF is a new one and a fingerprint bin is created for the segment. A new Bin Address Table

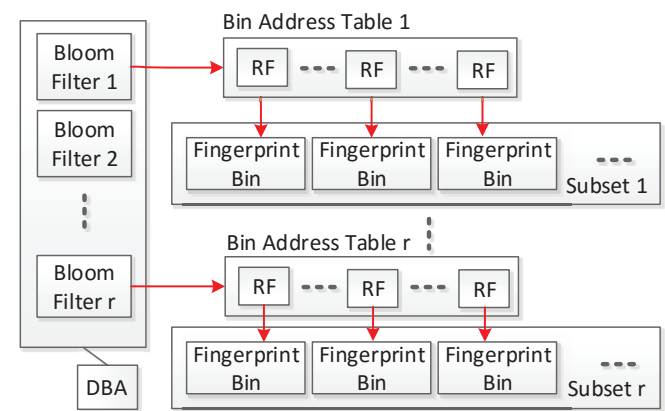

Figure 8: The organization of Bin Address Table. Each bloom filter in the DBA is responsible for a subset of RFs and each RF corresponds to a fingerprint bin containing fingerprints from resemblance segments. Knowing a RF, we can find the corresponding fingerprint bin if it exists.

$R F=[5, a, 4, b, 3, c, 2, d, 1, e, 9, e, 8, b, 6,5,8, c, 4,7]$

$$
\begin{array}{r}
\begin{array}{r}
{[5, a, 4, b, 3, c, 2, d, 1, e]} \\
\text { XOR } \quad[9, e, 8, b, 6,5,8, c, 4,7]
\end{array} \\
\text { Compact_RF }=[c, 4, c, 0,5,9, a, 1,5,9]
\end{array}
$$

Figure 10: A compacted RF resulting from the XOR operation between the first part 10 bytes and the second part 10 bytes.

entry associating the RF with the address of the newly created bin is inserted into the Bin Address Table. If the DBA acknowledges the existence of the RF, then RMD further searches for the RF in the Bin Address Table to verify its existence. Therefore, the Bin Address Table serves triple purposes of querying RF, finding the addresses of the fingerprint bin belonging to existing RFs and remedying the "false positive" answers from the DBA.

The Bin Address Table is kept in RAM to improve query performance. Suppose the system uses SHA1 fingerprint which takes 20 bytes, it may consume a lot of precious memory resources to accommodate the Bin Address Table. We make an optimization to the storage of Bin Address Table to halve the memory consumption. Specifically, we store RFs in a compact way. We divide a 20-byte RF into two 10-byte parts and store the XORed result of the two 10-byte subfingerprints as shown in Fig. 10.

\subsection{Fingerprint Bin}

In RMD, a Fingerprint Bin contains a collection of fingerprints from resemblance segments identified by the resemblance algorithm and it is also the on-disk fingerprint storage unit. Each entry in the bin contains a chunk fingerprint, the chunk size and physical location 


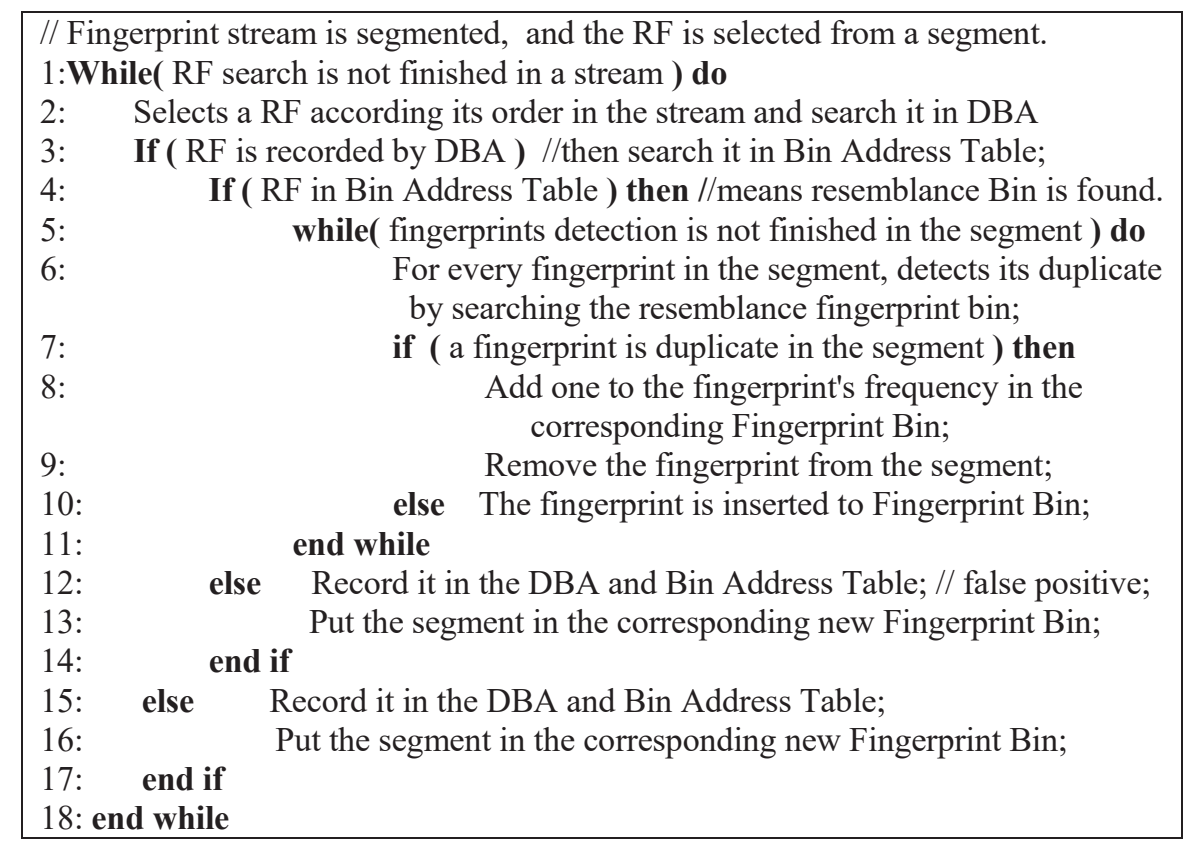

Figure 9: The pseudo-code of RMD workflow.

where the chunk is stored. Each fingerprint bin has an associated RF and when its RF is hit in the Bin Address Table, the entire fingerprint bin is brought in the memory and buffered in the fingerprint bin buffer for subsequent fingerprint comparisons. When a segment with the RF $f$ enters into the system, it is deduplicated against the fingerprints bin which is associated with the RF $f$. After eliminating duplicate fingerprints, the remaining new fingerprints in the segment are inserted into the fingerprint bin. Fingerprint bins are dynamically created in order to accommodate new segments.

\subsection{Frequency-based Fingerprint Retention Policy}

As the system ages, the amount of fingerprints in a fingerprint bin accumulates and may take up huge amount of storage space, thus affecting performance. In order to deal with this situation, RMD takes advantage of the frequency characteristic to manage the bin space. Fig. 2 shows that the capacity of a fingerprint bin can be limited as resemble segments accumulate in the fingerprint bin. Due to the most likely duplicate fingerprints being retained in the fingerprint bins and the skewed distribution of fingerprint frequency (see Fig. 3), the deduplication efficiency is well-maintained. Fingerprints in the same bin are sorted according to the frequency, and if the capacity exceeds a threshold, RMD cleans up those that have stayed the longest time and have the least frequency (most likely unique fingerprints). In this way, RMD minimizes the possibility of potentially important fingerprints being pruned prematurely. In order to keep potentially important fingerprints, the fingerprint bins can be set to different capacities dynamically, to account for the fact that each $\mathrm{RF}$ may correspond to various amounts of resemble segments. RMD ensures that each fingerprint bin is stored continuously on the disk so that only one disk IO is required to fetch the fingerprint bin into memory in its entirety. Meanwhile, it also prevents the fingerprint bin capacity from aggressively growing and thus causing performance decline.

\subsection{Buffer Mechanisms}

As shown in Fig. 5, to improve fingerprint query performance, RMD employs two buffers, namely $F p$ BinBuffer and RAM-Hit-Table. The FpBinBuffer buffer contains part of fingerprint bins for fingerprint comparisons, as it is unrealistic to store all fingerprints in memory. When a RF is found in the Bin Address Table, the corresponding fingerprint bin is brought into the FpBinBuffer buffer. Similarly, when a RF does not exist, then a new fingerprint bin is created in the FpBinBuffer. When replaced out, both newly created fingerprint bins and dirty fingerprint bins caused by fingerprint mergence are written back to the disk. In the meanwhile, RMD writes the fingerprint bins which are oversize to new locations to guarantee continuous 
storage of fingerprint bins. The old fingerprint bins are reclaimed at a later time by a garbage collection process. The RAM-Hit-Table provides a method to quickly determine whether a given fingerprint bin is currently buffered in the FpBinBuffer or not. Every time a new fingerprint bin is created in the buffer or an existing fingerprint bin is brought in from the disk, the RAM-Hit-Table is appropriately updated to reflect the changes. The RAM-Hit-Table also contains the LRU information about the buffered fingerprint bins to help make LRU-based replacement decisions.

\subsection{Fingerprint Index Workflow}

Summarizing the above discussions, the primary workflow of the fingerprint index in RMD is as below :

1. A fingerprint stream is cut into a sequence of segments with each segment containing the same amount of fingerprints and a RF is selected from each segment using the predefined sampling policy.

2. For each segment, RMD queries the DBA to check whether its RF exists in the Bin Address Table or not.

3. If the RF has not been recorded previously in the DBA or the RF has been recorded in the DBA but is not found to exist in the Bin Address Table, then the segment is considered as a new one. The $\mathrm{RF}$ is recorded in the DBA and then inserted to the BATable, and a fingerprint bin is created in the FpBinBuffer. Meanwhile, the RAM-Hit-Table is also updated correspondingly. Fingerprints are deduplicated within the same segment, and after that the remaining unique fingerprints are inserted in the fingerprint bin.

4. If the RF has been recorded in the DBA and is also found to exist in the Bin Address Table, then the fingerprint bin corresponding to the RF is brought in the FpBinBuffer buffer if it was not in the buffer. Meanwhile, the RAM-Hit-Table is also updated correspondingly. Then duplicate fingerprints in the segment are eliminated, and new fingerprints are inserted in the fingerprint bin.

5. When any fingerprint bin is replaced out from the memory, it is written to a new disk location if it is overloaded or if it is a newly created fingerprint bin. Otherwise, it is written back to the original location.

\subsection{Scalability Discussion}

It has been proved that DBA has good scalability and outstanding query performance (Wei et al., 2014).

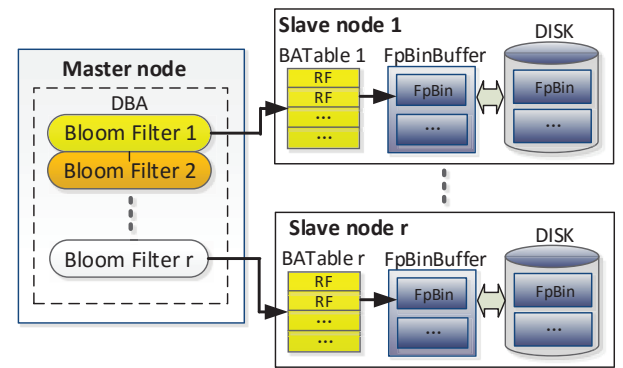

Figure 11: RMD can also be deployed in multi-node environments, providing good scalability. DBA resides on a master node, while Bin Address Table and fingerprint buffer are located on other slave nodes.

Our RMD leverages DBA, which ensures its good scalability. To facilitate the discussion, we qualitatively compare RMD with another scalable deduplication approach named MAD2 (Jiansheng et al., 2010), which also uses DBA for fingerprint query. MAD2 is an exact deduplication approach and the DBA is used to represent the membership of all fingerprints. By contrast, RMD uses DBA to only represent the membership of RFs. From the previous descriptions, we know that each fingerprint segment has only one RF, resulting in a much smaller DBA. Assuming there are $w$ fingerprints totally in each segment, the ratio between RF and the whole set of fingerprints is $1: w$, which means RMD can support $w$ times fingerprint capacity that of MAD2 with the same DBA. Equally said, the scalability of RMD is $w$ times better than MAD2 when the system expands. Furthermore, RMD adopts the frequencybased retention policy, which reduces the size of fingerprint bins, without compromising the deduplication ratio significantly. Finally, RMD can also be deployed in multi-node environments, as shown in Fig. 11. In this situation, the DBA is hosted on a master node, while the Bin Address Table and Fingerprint bins reside on other slave nodes.

\section{Evaluation}

\subsection{Experimental Environment}

In order to evaluate the feasibility and effectiveness of RMD, we have built a prototype system of RMD which takes fingerprint streams as its input. We have collected two public real world fingerprint trace sets and a dataset representing daily lab usage. These datasets imitate the typical scenarios of data deduplication in deployment, that is, data backup. We compare RMD with three other deduplication schemes: MAD2 (Jiansheng et al., 2010), SiLo (Xia et al., 2015) and Extreme Binning 
(Bhagwat et al., 2009) in respects of performance, deduplication efficiency, and RAM usage. Before our experiments, the dataset is processed by content-defined chunking (CDC) algorithm to generate fingerprints, which are then stored in files. RMD loads fingerprint streams from files and performs deduplication. Table 2 shows the key characteristics of the three datasets. The user-set and MAC-set datasets are collected from the website, http://tracer.filesystems.org (For more details of the two public datasets, please refer to their descriptions on the website.) (Tarasov et al., 2012), and the server-backup is collected from the machine snapshots in our lab. The user-set contains 18 snapshots of user directory spanning over nine months. The MAC-set contains 19 weekly snapshots starting from December 1 2013. The server-backup dataset was collected from monthly snapshots spanning a period of six months. All evaluations are carried out on an IBM server (system X 3620 M3) which features 8GB RAM, four-core $2.13 \mathrm{GHz}$ Intel Xeon E5606 and a RAID-5 storage system composed of 8 disk drivers.

In the process of exploring and implementing, we found that changes of the segment cardinality (the number of fingerprints in a segment) and bin capacity can have dramatic impacts on performance and efficiency. Therefore, we perform two categories of experiments. The first category is a sensitivity study on several key parameters, i.e., adjusting the different test parameters to search the parameters that lead to optimal performance and deduplication efficiency. The varying parameters include the segment cardinality, the mergence cardinality (the number of fingerprints which are absorbed to a bin). We omit the parameter exploration of DBA, as it has been extensively studied in (Wei et al., 2014). The second category is a comprehensive comparison between RMD and several other major deduplication approaches. In order to compare the fingerprint query efficiency among different schemes, we define a new metric called Found Deduplication Ratio (FDR), which indicates the ratio of detected duplicate fingerprints to the amount of actually existing duplicate fingerprints. (Comparing to deduplication ratio, FDR can more accurately reflect the ability of discovering duplicate chunks of different near-exact deduplication schemes). In the following subsections, we report and discuss the evaluation results.

\subsection{Parameter Exploration}

\subsubsection{Segment Cardinality and Mergence Cardinality}

In this subsection, we examine the performance impact of segment cardinality and mergence cardinality of
Fingerprint Bin. In our test, the frequency-based capacity limit strategy is adopted (set mergence cardinality to limit fingerprint bin capacity). Fig. 12 demonstrates the query throughput results of the three datasets. In each subfigure, the $\mathrm{x}$-axis represents the segment cardinality, the $y$-axis denotes the average query throughput. The curves indicate that the query performance varies with the segment cardinality and mergence cardinality of Fingerprint Bin. The mergence cardinality means the maximal number of new fingerprints which are inserted into one fingerprint bin. Full mergence means all fingerprints from different resemblance segments are allowed to be merged into the corresponding fingerprint bin without enforcing limit capacity. The maximal size of a fingerprint bin is equal to the segment cardinality plus mergence cardinality.

The results of the three different datasets have shown similar tendency. When the segment cardinality is set to 128 , RMD delivers a peak performance for all the three datasets across all mergence cardinalities, reaching up to 1.5 million fingerprints per second. Generally, when the segment cardinality is less than 128 , the query performance increases with the segment cardinality. When the segment cardinality becomes larger than 128 , the query performance decreases as the cardinality increases. The reason is because when the segment cardinality is smaller, more segments and RFs have to be processed. On the contrary, if the segment cardinality is too big, it implies more fingerprints in a segment need to be searched in the fingerprint bins.

Next, let's take a look at the impact of mergence cardinality on the query performance. As it is shown in Fig. 12, when the mergence cardinality ranges from 0 to 1024, the maximal performance gap is only 0.23 million fingerprints per second. This indicates that when the frequency-based capacity limit strategy is enforced, different mergence cardinalities show similar query performance. The increase of the mergence cardinality does not noticeably affect the fingerprint retrieval performance. Additionally, we can observe that the query performance of the full mergence is significantly lower than the other mergence cardinality scenarios, as the exhaustive merging operations introduce non-trivial overhead. In addition, when the segment cardinality is small, the performance gap between full mergence strategy and other frequency-based capacity limit strategy is large, but when the segment cardinality increases, the gap becomes narrower. This is mainly because a larger mergence cardinality results in larger fingerprint bins, which in turn impacts the query efficiency. When the segment cardinality is large, the performance advantages of frequency-based capacity 
Table 2: Features of the used datasets

\begin{tabular}{rrrr}
\hline & user-set & MAC-set & server-backup \\
\hline Total size & $5.88 \mathrm{~TB}$ & $3.71 \mathrm{~TB}$ & $1.74 \mathrm{~TB}$ \\
Average file size & $120.7 \mathrm{~KB}$ & $64.2 \mathrm{~KB}$ & $151.7 \mathrm{~KB}$ \\
Average chunk size & $4.67 \mathrm{~KB}$ & $4.29 \mathrm{~KB}$ & $2.95 \mathrm{~KB}$ \\
Exact-Deduplication ratio & 25.20 & 33.00 & 10.25 \\
\hline
\end{tabular}

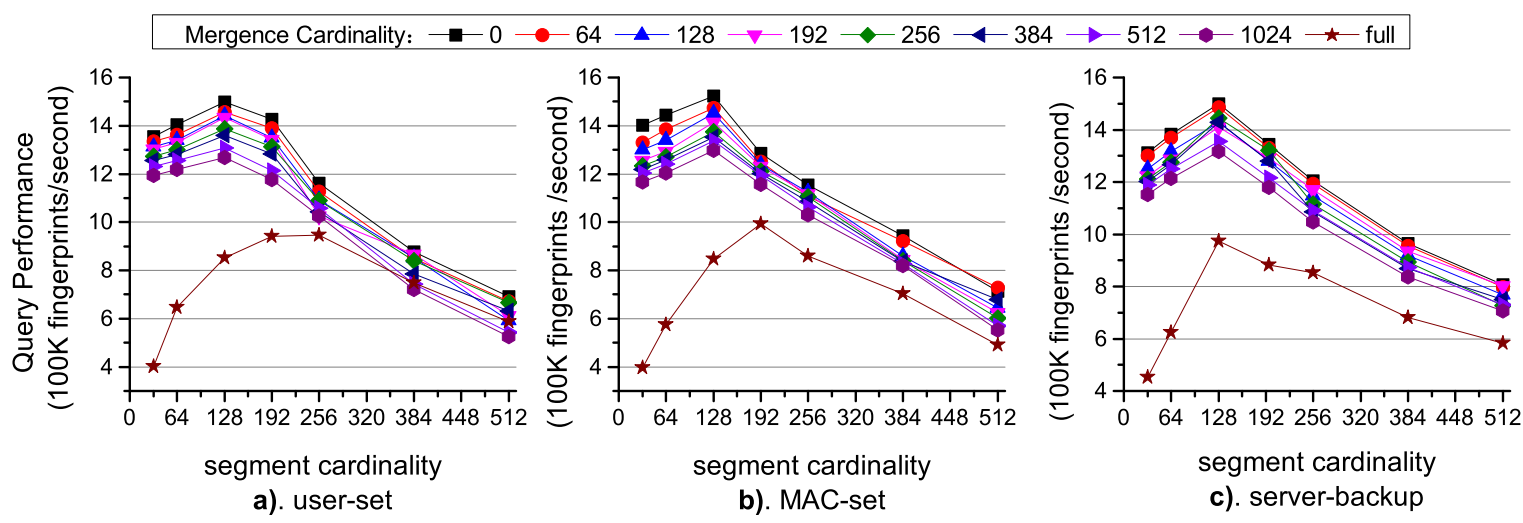

Figure 12: The query performance of the three datasets with varying segment cardinality and mergence cardinality. The x-axis denotes the segment cardinality and the y-axis denotes query throughput. Different curves represent the performance of different mergence cardinalities. Generally, a segment cardinality of 128 delivers the peak throughput. The 0 curve means no mergence is allowed.

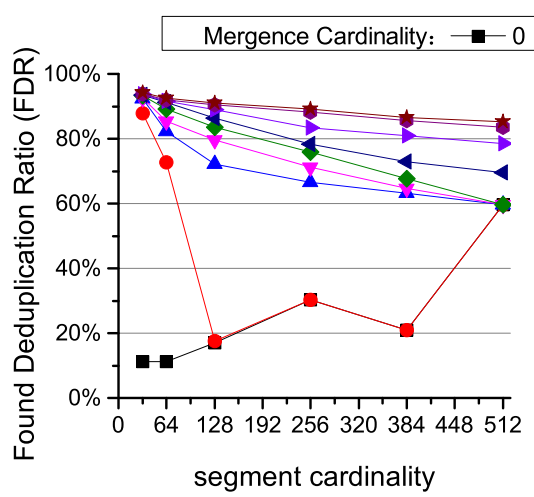

a). user-set

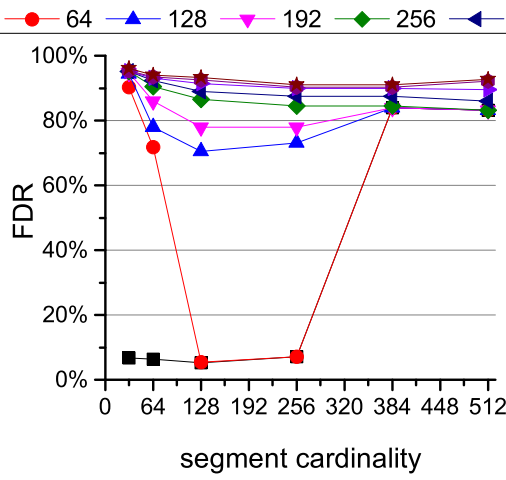

b). MAC-set

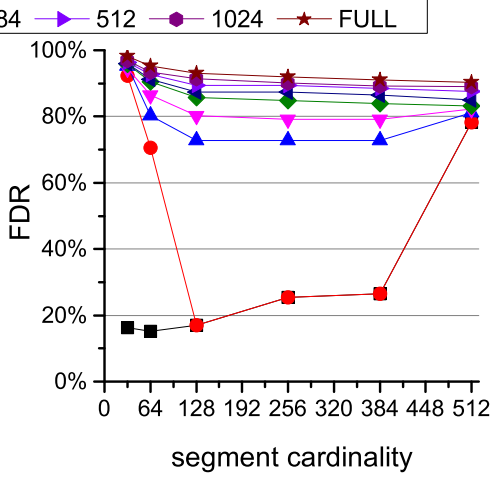

c). server-backup

Figure 13: The trend of Found Deduplication Ratio (FDR) values with varying segment cardinality and mergence cardinality. Generally, given a certain value of mergence cardinality, a relatively small segment cardinality will result in a higher FDR, i.e., better deduplication ratio. The 0 curve means no mergence is allowed. 
limit strategy is weakened. But on the whole, the frequency-based capacity limit strategy can effectively reduce the number of fingerprint queries, thus improving performance.

We have also investigated the impacts of segment cardinality and mergence cardinality on the deduplication ratio using the metric of $F D R$. Fig. 13 shows the results. From the figure, we can obtain several conclusions. First, when the mergence cardinality is small, the Found Deduplication Ratios (FDRs) exhibit remarkably large fluctuations. When the segment cardinality is between 128 and 256, and the mergence cardinality is less than 64, the values of FDR are quite small. The minimal value occurs to the MAC dataset and is only $6.0 \%$. Meanwhile, there are some overlapping points between curve- 0 and curve- 64 which means they have the same FDRs with smaller mergence cardinality and bigger segment cardinality. But with a mergence cardinality larger than 128, the FDR shows a consistent trend, i.e., they are closer to each other. Moreover, with any specific mergence cardinality value greater than 256, the FDR decreases with the segment cardinality. The reason is that with larger segment cardinalities, the probability of dropping off duplicate fingerprint increases, degrading deduplication efficiency. Second, as the mergence cardinality becomes larger, the reward in FDR becomes smaller. In the extreme case, we can see that the curve-1024 nearly overlaps with the curvefull. Finally, based on the results, we conclude a general guidance of getting the best FDRs. Given a certain value of mergence cardinality, a smaller segment cardinality is preferred. With a smaller segement, the accuracy of finding duplicate fingerprints via the same RF is improved. In the extreme case in which the segment cardinality is 1 , RMD evolves to exact deduplication.

\subsubsection{Trend of Found Deduplication Ratio (FDR)}

In this section, we investigate the relationship between the resemblance mergence policy and the trend of FDR. In previous tests, we report the FDR as the averaged values across all the snapshots within the same datasets. It does not reveal the FDR change trend as fingerprints accumulate. We present a microscopic view of the changing trend of FDR of the different snapshots within the same datasets. In this evaluation, we have performed tests with two datasets, MAC-set and userset, and the segment cardinality is set to $64,128,512$ respectively. Figure 14 gives the results of FDR trend in two cases where resemblance mergence strategy is enabled and disabled, respectively. From the figure, we can see that the mergence policy has a significant impact on the FDR, especially the mergence cardinality is set to
1024. In this case, although the FDR is pretty low at the beginning, ranging from $40 \%$ to $50 \%$, as fingerprints accumulate it quickly increases to $96.8 \%$ at the third snapshot. After that, it persistently remains at the level between $96 \%$ and $98 \%$, with the exception of the $13^{\text {th }}$ snapshot of the user-set dataset. We found that the sharp decline was caused by a user switch, which means starting from the $13^{\text {th }}$ snapshot, the dataset belongs to a different user and has very different content. With non-mergence policy, the majority of FDRs are only around $10 \%$ when the segment cardinality is set 64 or 128. Although the FDR shows a slightly increasing trend as fingerprints accumulate, the maximum value is still less than $22 \%$. In order to search for the best possible results with non-mergence strategy, we change the values of segment cardinality and make further comparisons with mergence strategy for the MAC-set and user-set dataset. Figure 15 shows the comparison results. For the non-mergence strategy, when segment cardinality is below 512, the FDR is low. When segment cardinality approaches 512 , the FDR reaches the maximum value, which is $84 \%$. However, when the segment cardinality becomes greater than 512, the FDR begins to fall. At the same time, the query performance decreases rapidly as segment cardinality increases. This means when adopting the non-mergence strategy, a segment cardinality of 512 generates the best results. This observation is consistent with the conclusions of SiLo (Xia et al., 2015). In general, comparing with the optimal cases, the FDR of the non-mergence strategy is $15 \%$ less than that of the mergence strategy, and the query performance of non-mergence strategy is only half that of the latter. That implies mergence policy does help improve deduplication efficiency.

\subsection{Comparative Evaluations}

This subsection presents comparison results between RMD and three other approaches, namely MAD2 (Jiansheng et al., 2010), SiLo (Xia et al., 2015), and Extreme binning (Bhagwat et al., 2009). The parameters of RMD are set as following: the mergence cardinality is 1024 with frequency-based capacity limit strategy, segment size is 64 , the DBA contains 128 bloom filters at maximum, and each group of bloom filter contains 64 bloom filters. The false positive rate of each bloom filter is ensured to be below $10^{-5}$. The MAD2 (Jiansheng et al., 2010) are set as following: the DBA contains 128 bloom filters at maximum, and each group of bloom filter contains 64 bloom filters. The false positive rate of each bloom filter is also ensured to be below $10^{-5}$. The Hash Bucket Matrix (HBM), of which each row contains 1024 hash buckets, 


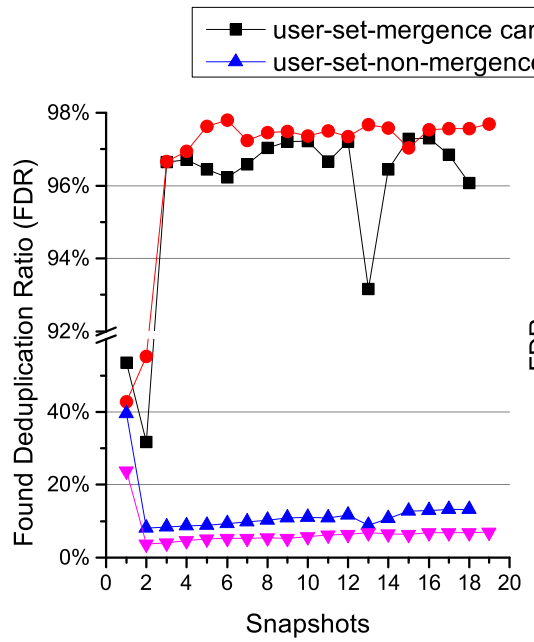

a) segment cardinality $=64$

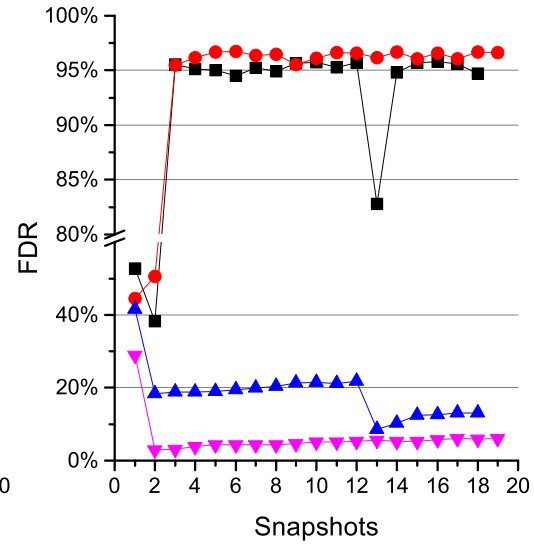

b) segment cardinality $=128$

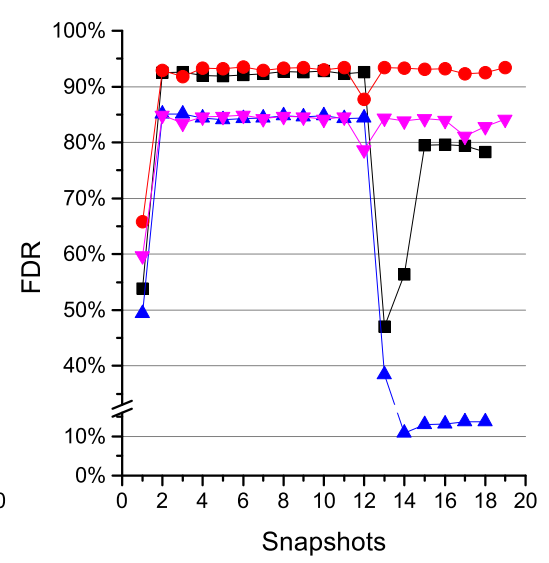

c) segment cardinality $=512$

Figure 14: The trend of Found Deduplication Ratio (FDR) as datesets accumulate. The segment cardinality is set to 64, 128, 512 respectively. Each point in the curves represents the FDR of the corresponding snapshot. Overall, the FDR of mergence policy is consistently much higher than that of non-mergence policy.

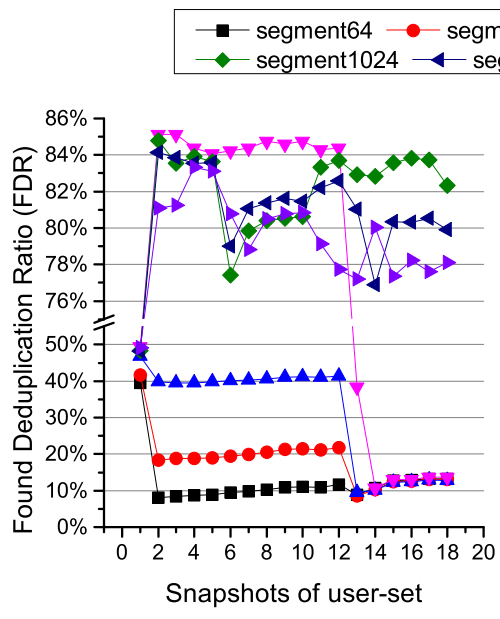

a)

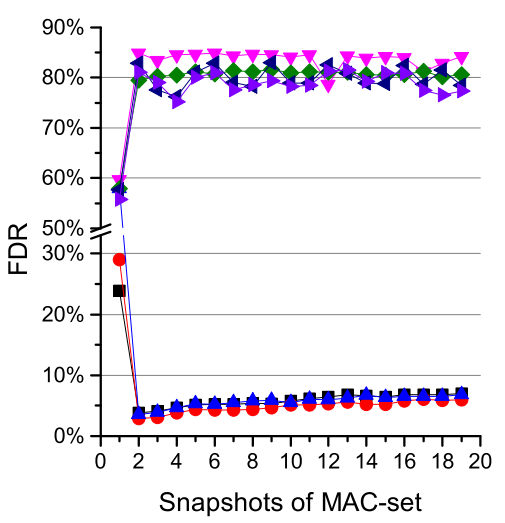

b)

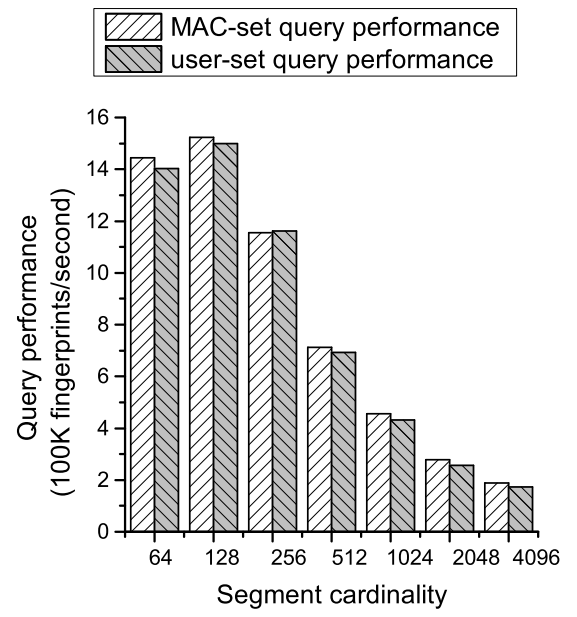

c)

Figure 15: The Found Deduplication Ratio (FDR) with non-mergence strategy as the segment cardinality changes for the user-set and MAC-set dataset. Setting a segment cardinality of 512 results in the best FDR. There is an exception of the $13^{\text {th }}$ snapshot of the $u s e r$-set dataset. We found that the sharp decline was caused by a user switch, meaning starting from the $13^{\text {th }}$ snapshot, the dataset belongs to a different user and has very different content. 


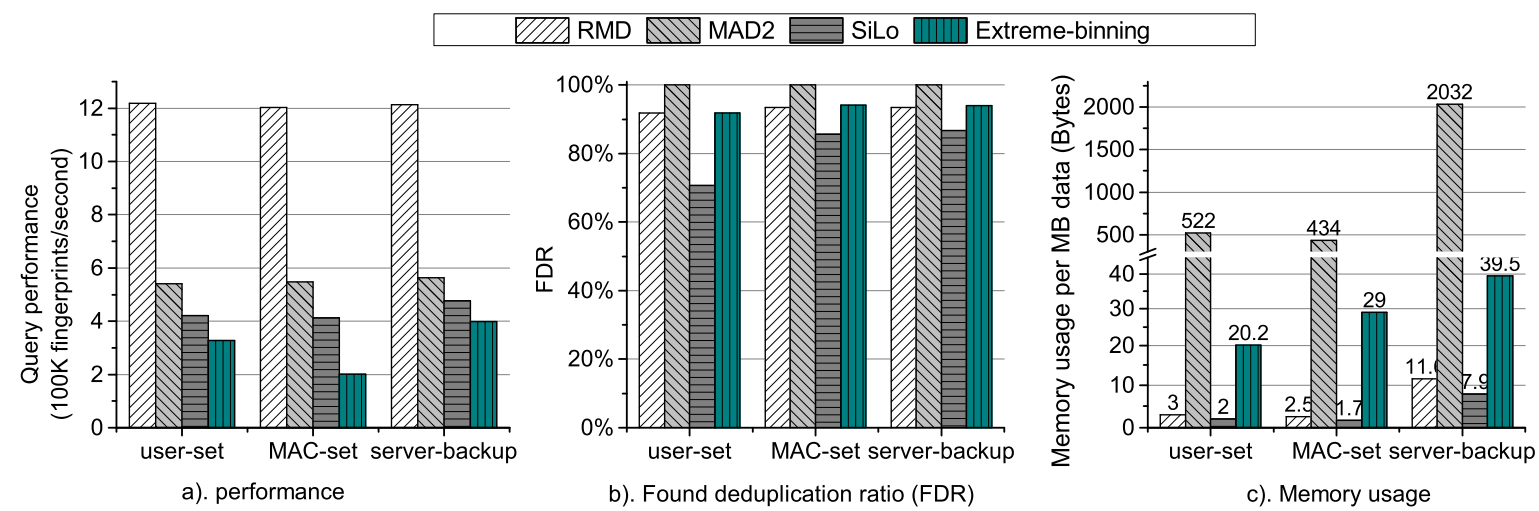

Figure 16: The figure compares the query performance (subfig a), the FDR (subfig b) and the RAM usage (subfig c) of RMD with other approaches. RMD delivers much higher query performance, while still providing comparable deduplication efficiency. In fact, it improves deduplication ratio as well in most scenarios.

and each bucket can store 1024 fingerprint records. The Extreme binning setting is set to be the default mode as in (Bhagwat et al., 2009) (no parameters). We configure the SiLo system as SiLo-2MB (Xia et al., 2015) with block size being $2 \mathrm{MB}$. We compare their query performance, FDR, and RAM consumption. From the experimental analysis of published papers we can see that these parameter settings of compared schemes represent the best settings for their systems.

\subsubsection{Query Performance}

Fig. 16a compares the query throughput of the four deduplication approaches. As demonstrated in the figure, RMD has the best performance for all the three datatsets, reaching an average throughput of 1.2 MFPS. MAD2 wins the second place, achieving an average throughput of 0.55 MFPS, which is only $45.8 \%$ of RMD. The benefits of RMD and MAD2 are mainly attributed to the deployment of high-performance DBA. SiLo achieves an average throughput of 0.44 MFPS, which is $36.6 \%$ of RMD. The Extreme Binning performs the worst, delivering an average throughput of 0.31 MFPS. We attribute the reason to its inherent shortcoming, i.e., it uses a file as the segment unit, which can cause unbalanced amounts of fingerprints in different bins, thus affecting query performance.

\subsection{2. $F D R$}

Fig. 16b shows the comparison of FDR among the four deduplication schemes. As illustrated, the average FDR of MAD2 is $100 \%$, because MAD2 is an exact deduplication scheme, which eliminates all duplicate fingerprints. The FDR of RMD is always above $90 \%$ across the three datasets, while SiLo has a slightly lower FDR than RMD, but it still has a very high FDR. Extreme binning performs comparatively with RMD and reaches an average of 93.3\% FDR. This set of results, together with the results in the preceding section have shown that RMD can deliver much better query performance, while still providing comparable deduplication efficiency. In fact, in most scenarios, it improves deduplication ratio at the same time relative to existing approaches.

\subsubsection{RAM Consumption}

To compare RAM usage, we use the same method as in the SiLo (Xia et al., 2015) to calculate memory consumption. Figure $16 \mathrm{c}$ shows the memory usage of the four deduplication schemes. Because the fingerprint values are homogeneously distributed in the entire hash space, each fingerprint subset needs to establish an index in memory and all fingerprints need to be buffered in memory, which cause the memory usage of MAD2 relatively large for every dataset. The memory usage of Extreme binning mainly comes from storing representative fingerprints. When the dataset is composed of many small files or the average chunk size is set relatively small, the memory usage becomes relatively large, evidenced by the MAC-set and server-backup datasets. SiLo has the lowest memory overhead, which mainly results from its relatively large block size. Due to the relative small segment cardinality and compressed RF, RMD embraces slightly higher memory overhead than that of SiLo. However, as the segment cardinality changes from 64 to 128 , the memory overhead drops to a half, while the query performance increases by an average of $6.8 \%$ and the FDR decreases by $1.5 \%$. 


\section{Related Work}

With the explosive growth of datasets, fingerprint indexing in deduplication systems becomes increasingly challenging and has attracted a lot of research interest. DDFS (Zhu et al., 2008) is one of the early schemes to exploit backup data spatial locality and cache prefetching scheme to effectively improve data query efficiency and decrease disk accesses caused by disk index. In addition, DDFS uses the Bloom filter to boost fingerprint index performance. Chunkstash (Debnath et al., 2010) improves the fingerprint index performance by utilizing uniform chunk sampling, spatial locality, and high performance flash memory. Resemblance data detection technique (Kulkarni et al., 2004) uses the resemblance based theory (Broder, 2000) to detect duplicate data. In order to detect the resemblance of two datasets, it first splits data streams into different segments each of which contains many chunks, and then chooses a representative chunk from each segment. It determines resemblance segments by comparing their representative chunks. If two representative chunks are the same, then the two corresponding segments are assumed to resemble and they are deduplicated against each other. Several existing works have successfully leveraged the resemblance detention technique. Sparse indexing (Lillibridge et al., 2009) utilizes a sampling strategy to select a representative fingerprint in each segment and chooses deduplication segment candidates based on the resemblance algorithm. Moreover, it employs multisegment prefetching strategy to realize duplicate data retrieval. This approach can reduce memory usage effectively, and multi-segment prefetching can increase data deduplication ratio (Fu et al., 2015). However, the prefetching of resemblance segment requires multiple alignment, which increases the retrieval cost. Extreme Binning (Bhagwat et al., 2009) is another example which exploits sampling strategy for deduplication. In the system, a file is used as the basic resemblance unit, and thus it is more suitable for datasets mainly containing small-sized files. With datasets containing large-sized files, the deduplication ratio would decline quickly.

As a highly space-efficient random data structure, Bloom filter (Broder and Mitzenmacher, 2004) is able to effectively improve index query performance and decrease RAM consumption at the same time. It has been widely used in large scale distributed storage systems such as Google's BigTable (Chang et al., 2008) and Data Domain's DDFS (Zhu et al., 2008). Although Bloom Filter is efficient in representing static sets, it faces challenges when dealing with dynamic sets. Xie et al. (Xie et al., 2007) build a scalable Bloom Filter Array which can dynamically expand its capacity by creating new bloom filters. Almeida et al. (Almeida et al., 2007) design another scalable Bloom filter array through increasing capacity of single bloom filter and diminishing its false positive ratio. Compared with Xie's scheme, Almeida's approach is more flexible in controlling the false positive ratio. But Almeida's scheme needs more hash functions because of the usage of heterogeneous bloom filters, which may increase the computational overhead. Hao et al. (Hao et al., 2008) propose a fill-ratio-restricted Bloom filter array to expand query capacity, which minimizes the space consumption. DBA (Wei et al., 2014) is proposed to represent membership for variable large datasets in storage systems. DBA consists of groups of homogeneous Bloom filters that can be created dynamically as needed and it provides excellent retrieval performance and good scalability. MAD2 (Jiansheng et al., 2010) exploits DBA algorithm to build a high throughput and scalable deduplication system. RMD also leverages the salient features of DBA to speed up the process of locating the segment bins given a RF. Deduplication has also been recently researched in flash-based storage systems to reduce flash write traffic and thus improve flash lifespan (Chen and Shen, 2016b; Li et al., 2016).

\section{Conclusion}

Fast fingerprint index query is highly desired in deduplication systems, as it largely determines the overall deduplication performance. In this paper, we present RMD, a fast deduplication approach to speeding up the fingerprint index performance. RMD takes advantage of the resemblance algorithm and dynamic Bloom Filter Array (DBA) to greatly reduce the query range when performing data deduplication. The resemblance algorithm is used to cluster resemblance segments into the same bins and the DBA is used to facilitate the process of finding the right bin given a representative fingerprint. Due to the convenience of finding the right segment candidates enabled by the novel organization of binned segments, RMD delivers high fingerprint query performance. In addition, segment clustering helps find more segment candidates for fingerprint verification without causing additional IOs, thus improving deduplication efficiency. Evaluation results with realworld workloads have shown that RMD significantly improves fingerprint query performance, while reducing RAM requirements at the same time. 


\section{Acknowledgements}

This work was supported in part by the Natural Science Foundation of China (NSFC) under Grant Nos. 61502189 and 61232004, and the U.S. National Science Foundation (NSF) under Grant Nos. CCF-1547804, CNS-1218960, and CNS-1320349. Conclusions or recommendations expressed in this material are those of the author(s) and do not necessarily reflect the views of the funding agencies.

\section{References}

Almeida, P. S., Baquero, C., Preguiça, N., Hutchison, D., 2007. Scalable bloom filters. Information Processing Letters 101 (6), 255-261.

Amvrosiadis, G., Bhadkamkar, M., 2015. Identifying Trends in Enterprise Data Protection Systems. In: Proceedings of the 2015 USENIX Annual Technical(USENIX ATC'15). IEEE, pp. 151164.

Bhagwat, D., Eshghi, K., Long, D. D., Lillibridge, M., 2009. Extreme binning: Scalable, parallel deduplication for chunk-based file backup. In: MASCOTS'09. IEEE, pp. 1-9.

Bianco, G. D., Galante, R., Gon?alves, M. A., Canuto, S., Heuser, C. A., Sept 2015. A practical and effective sampling selection strategy for large scale deduplication. IEEE Transactions on Knowledge and Data Engineering 27 (9), 2305-2319.

Broder, A., Mitzenmacher, M., 2004. Network applications of bloom filters: A survey. Internet mathematics 1 (4), 485-509.

Broder, A. Z., 1997. On the resemblance and containment of documents. In: Compression and Complexity of Sequences 1997. Proceedings. IEEE, pp. 21-29.

Broder, A. Z., 2000. Identifying and filtering near-duplicate documents. In: Combinatorial pattern matching. Springer, pp. 110.

Chang, F., Dean, J., Ghemawat, S., Hsieh, W. C., Wallach, D. A., Burrows, M., Chandra, T., Fikes, A., Gruber, R. E., 2008. Bigtable: A distributed storage system for structured data. ACM Transactions on Computer Systems (TOCS) 26 (2), 4

Chen, Z., Shen, K., February 2016a. OrderMergeDedup: Efficient, Failure-Consistent Deduplication on Flash. In: FAST'16. IEEE, IEEE, Santa Clara, CA, pp. 151-164.

Chen, Z., Shen, K., 2016b. Ordermergededup: Efficient, failureconsistent deduplication on flash. In: 14th USENIX Conference on File and Storage Technologies (FAST 16). pp. 291-299.

Debnath, B., Sengupta, S., Li, J., 2010. Chunkstash: Speeding up inline storage deduplication using flash memory. In: Proceedings of the 2010 USENIX Conference on USENIX Annual Technical Conference. USENIXATC'10. USENIX Association, Berkeley, CA, USA, pp. 16-16.

Dong, W., Douglis, F., Li, K., Patterson, R. H., Reddy, S., Shilane, P., 2011. Tradeoffs in scalable data routing for deduplication clusters. In: FAST. pp. 15-29.

Fu, M., Feng, D., Hua, Y., He, X., Chen, Z., Xia, W., Zhang, Y., Tan, Y., 2015. Design tradeoffs for data deduplication performance in backup workloads. In: FAST'15. USENIX Association, Santa Clara, CA, pp. 331-344

Fu, Y., Jiang, H., Xiao, N., Tian, L., Liu, F., Xu, L., May 2014 Application-aware local-global source deduplication for cloud backup services of personal storage. IEEE Transactions on Parallel and Distributed Systems 25 (5), 1155-1165.
Guo, D., Wu, J., Chen, H., Luo, X., et al., 2006. Theory and network applications of dynamic bloom filters. In: INFOCOM. pp. 1-12.

Hao, F., Kodialam, M., Lakshman, T., 2008. Incremental bloom filters. In: INFOCOM 2008. The 27th IEEE Conference on Computer Communications. IEEE.

Jiansheng, W., Hong, J., Ke, Z., Dan, F., 2010. Mad2: A scalable high-throughput exact deduplication approach for network backup services. In: Mass Storage Systems and Technologies (MSST), 2010 IEEE 26th Symposium on. IEEE, pp. 1-14.

Kaczmarczyk, M., Dubnicki, C., May 2015. Reducing Fragmentation Impact with Forward Knowledge in Backup Systems with Deduplication. In: Proceedings of the $8^{\text {th }}$ International Systems and Storage Conference(SYSTOR'15). IEEE, Haifa, Israel, pp. 201-208.

Kruus, E., Ungureanu, C., Dubnicki, C., 2010. Bimodal content defined chunking for backup streams. In: FAST. pp. 239-252.

Kulkarni, P., Douglis, F., LaVoie, J. D., Tracey, J. M., 2004. Redundancy elimination within large collections of files. In: USENIX Annual Technical Conference, General Track. pp. 59-72.

Li, J., Li, Y. K., Chen, X., Lee, P. P. C., Lou, W., May 2015. A hybrid cloud approach for secure authorized deduplication. IEEE Transactions on Parallel and Distributed Systems 26 (5), 12061216.

Li, W., Jean-Baptise, G., Riveros, J., Narasimhan, G., Zhang, T., Zhao, M., 2016. Cachededup: in-line deduplication for flash caching. In: 14th USENIX Conference on File and Storage Technologies (FAST 16). pp. 301-314.

Li, Y.-K., Xu, M., Ng, C.-H., Lee, P. P. C., Dec. 2014. Efficient hybrid inline and out-of-line deduplication for backup storage. Trans. Storage 11 (1), 2:1-2:21.

Lillibridge, M., Eshghi, K., Bhagwat, D., Deolalikar, V., Trezis, G., Camble, P., 2009. Sparse indexing: Large scale, inline deduplication using sampling and locality. In: FAST. pp. 111-123.

Maltzahn, C., Molina-Estolano, E., Khurana, A., Nelson, A. J., Brandt, S. A., Weil, S., 2010. Ceph as a scalable alternative to the hadoop distributed file system. login: The USENIX Magazine 35, 38-49.

Mao, B., Jiang, H., Wu, S., Fu, Y., Tian, L., Mar. 2014. Readperformance optimization for deduplication-based storage systems in the cloud. Trans. Storage 10 (2), 6:1-6:22.

Min, J., Yoon, D., Won, Y., 2011. Efficient deduplication techniques for modern backup operation. Computers, IEEE Transactions on 60 (6), 824-840.

Quinlan, S., Dorward, S., 2002. Venti: A new approach to archival storage. In: FAST. pp. 89-101.

Shim, H., 2017. Phash: A memory-efficient, high-performance keyvalue store for large-scale data-intensive applications. Journal of Systems and Software 123, $33-44$.

Shvachko, K., Kuang, H., Radia, S., Chansler, R., 2010. The hadoop distributed file system. In: Mass Storage Systems and Technologies (MSST), 2010 IEEE 26th Symposium on. IEEE, pp. $1-10$.

Srinivasan, K., Bisson, T., Goodson, G. R., Voruganti, K., 2012. idedup: latency-aware, inline data deduplication for primary storage. In: FAST. pp. 1-14.

Tarasov, V., Mudrankit, A., Buik, W., Shilane, P., Kuenning, G., Zadok, E., 2012. Generating realistic datasets for deduplication analysis. In: USENIX Annual Technical Conference. pp. 261-272.

Wang, F., Wang, H., Li, J., Gao, H., 2013. Graph-based reference table construction to facilitate entity matching. Journal of Systems and Software 86 (6), 1679 - 1688.

Wei, J., Jiang, H., Zhou, K., Feng, D., 2014. Efficiently representing membership for variable large data sets. IEEE Trans. Parallel Distrib. Syst. 25 (4), 960-970.

Wildani, A., Miller, E. L., Rodeh, O., 2013. Hands: A heuristically 
arranged non-backup in-line deduplication system. In: Data Engineering (ICDE), 2013 IEEE 29th International Conference on.

Xia, W., Jiang, H., Feng, D., Hua, Y., April 2015. Similarity and locality based indexing for high performance data deduplication. IEEE Transactions on Computers 64 (4), 1162-1176.

Xie, K., Min, Y., Zhang, D., Wen, J., Xie, G., 2007. A scalable bloom filter for membership queries. In: Global Telecommunications Conference, 2007. GLOBECOM'07. IEEE, pp. 543-547.

Xu, J., Zhang, W., Zhang, Z., Wang, T., Huang, T., 2016. Clusteringbased acceleration for virtual machine image deduplication in the cloud environment. Journal of Systems and Software 121, 144 156.

Zhu, B., Li, K., Patterson, R. H., 2008. Avoiding the disk bottleneck in the data domain deduplication file system. In: FAST. pp. 1-14.

\section{Authors}

Panfeng Zhang is currently a Ph.D. student in the College of Computer Science and Technology at Huazhong University of Science and Technology (HUST), Wuhan, China. His main research interests include data storage systems, operating systems and key value systems, etc. (panf_zhang@hust.edu.cn)

Ping Huang received his Ph.D. degree from Huazhong University of Science and Technology in 2013. He is currently a Ph.D student in the Department of Computer and Information Sciences at Temple University, Philadelphia, PA. His main research interests include non-volatile memory, operating system, distributed systems, DRAM, GPU, key value systems, etc. He has published papers in various international conferences and journals, including SYSTOR, NAS, MSST, USENIX ATC, Eurosys, Performance, INFOCOM, SRDS, MASCOTS, Journal of Systems Architecture (JSA), Performance Evaluation (PEVA), IEEE Transactions on Parallel and Distributed Systems(TPDS), Sigmetrics, ICPP, etc. (templestorager@temple.edu)

Xubin He received the BS and MS degrees in computer science from Huazhong University of Science and Technology, China, in 1995 and 1997, respectively, and the $\mathrm{PhD}$ degree in electrical engineering from University of Rhode Island, Kingston, RI, in 2002. He is currently a professor in the Department of Computer and Information Sciences at Temple University. His research interests include computer architecture, data storage systems, virtualization, and high availability computing. Dr. He received the Ralph E. Powe Junior Faculty Enhancement Award in 2004 and the Sigma Xi Research Award (TTU Chapter) in 2005 and 2010. He is a senior member of the IEEE, a member of the IEEE Computer Society and USENIX. (xubin.he@temple.edu)

Hua Wang received the Ph.D. degree from the College of Computer Science and Technology, Huazhong University of Science and Technology (HUST) in 2009. Currently, she is a lecturer of Wuhan National Laboratory for Optoelectronics at HUST. Her main research interests include computer architecture, cloud storage system and service. (hwang@hust.edu.cn)

Ke Zhou received the Ph.D. degree from the College of Computer Science and Technology, Huazhong University of Science and Technology (HUST) in 2003. Currently, he is a professor of the College of Computer Science and Technology at HUST. His main research interests include computer architecture, network storage systems, parallel I/O, storage security and network data behavior theory. (k.zhou@hust.edu.cn). 